\title{
On the various impact of chemical composition and elastic strain in SiGe nanoscale islands to the diffuse X-ray scattering
}

\author{
M. Hanke ${ }^{\mathrm{a}, *}$, T. Boeck ${ }^{\mathrm{b}}$ \\ ${ }^{a}$ Martin-Luther-Universität Halle-Wittenberg, Fachbereich Physik, Hoher Weg 8, D-06120 Halle/Saale, Germany \\ ${ }^{\mathrm{b}}$ Institut für Kristallzüchtung, Max-Born-Straße 2, D-12489 Berlin, Germany
}

Available online 25 January 2006

\begin{abstract}
High-resolution X-ray diffraction in combination with kinematical scattering simulations based on finite element calculations for the elastic strain field has been utilized to investigate the chemical composition of $\mathrm{SiGe} / \mathrm{Si}\left(\begin{array}{lll}0 & 0 & 1\end{array}\right) \mathrm{Stranski-Krastanov}$ islands. Therefore, the intensity distribution in the vicinity of the symmetric $(004)$ reflection has been three dimensionally measured and compared with respective simulations. Around an asymmetrical reflection, e.g. $\mathrm{Si}(113)$, the impact of lateral and vertical strain tensor components on the diffraction pattern decouples. This allows to distinguish between tetragonally distorted regions at the island bottom and elastically relaxed unit cells of cubic shape at the island apex in accordance with finite element calculations. Artificially switching off and on the various scattering abilities of silicon and germanium in otherwise equivalent simulations yields practically no difference in the resulting scattering. Thus, at X-ray energies far from absorption edges, the diffuse scattering is dominated by elastic strain which is caused by the chemical profile.
\end{abstract}

(C) 2006 Elsevier B.V. All rights reserved.

PACS: 68.65.-k; 61.10.-i; 81.15.Lm

Keywords: X-ray scattering; $\mathrm{SiGe/Si}$; Finite element method

\section{Introduction}

For a large variety of material combinations the Stranski-Krastanov growth mode provides an elegantly simple way to self-form and further self-assemble highly ordered arrays of low-dimensional structures, e.g. Ref. [1] The strain energy which will be initially accumulated during the heteroepitaxy of the very first amount of material can be accommodated in the crystal lattice resulting in a two-dimensional layer growth. However, after a critical thickness, depending on the actual lattice mismatch, the formation of three-dimensional islands is achieved, which becomes energetically favorable instead of further layer growth. Thus, the energy gain due to elastic relaxation overbalances the additional free surface energy. Moreover, in a near-equilibrium growth environment the final island size inversely scales with the accommodated

\footnotetext{
${ }^{*}$ Corresponding author.

E-mail address: hanke@physik.uni-halle.de (M. Hanke).
}

strain energy [2]. Liquid phase epitaxy (LPE) of $\mathrm{SiGe} / \mathrm{Si}$ serves as a suitable model system to achieve a more comprehensive understanding of the observed phenomena and the underlying mechanisms. In a complementary way to direct analytical techniques highly resolved X-ray scattering techniques can provide statistically relevant information concerning elastic strain and morphology, e.g. Refs. [3-5].

\section{Experiment}

LPE operates very close to thermodynamical equilibrium and hence yields a near-equilibrium island morphology. In case of $\mathrm{SiGe} / \mathrm{Si}(001)$ it consists of truncated pyramids made of four adjacent $\left\{\begin{array}{lll}1 & 1 & 1\end{array}\right\}$ and a single $\left(\begin{array}{lll}0 & 0 & 1\end{array}\right)$ top facet [6]. An averaged island base of $130 \mathrm{~nm}$ along a mean island height of $65 \mathrm{~nm}$ yields a geometric aspect ratio base vs. height of two. Moreover, the islands self-assemble in a strain-driven process into extended chains along the elastically soft $\langle 100\rangle$ directions [7]. 
Naturally scattering from nanoscale objects is expected to appear rather weak in contrast to bulky objects. Moreover, the smaller the objects the more extended the diffuse intensity in reciprocal space whose detection generally requires highly brilliant X-ray sources. Thus, the measurements we present here have been performed at the BW2 synchrotron station at HASYLAB, Hamburg using an X-ray energy of $8 \mathrm{keV}$. A positional-sensitive detector (PSD) placed in a distance of $1000 \mathrm{~mm}$ from the sample position records the diffusely scattered intensity along a (curved) line in reciprocal space. Either by rocking the sample $(\Omega$-scan) or moving sample and detector in a certain ratio $(\Omega / 2 \Theta$-scan) two-dimensional intensity distributions were recorded. Thereby, the entrance slit $(1 \mathrm{~mm})$ in front of the PSD restricts the resolution perpendicular to the scattering plane to about $0.006 \AA^{-1}$.

In order to quantitatively evaluate the measured distributions we have performed kinematical scattering simulations based on a two-step iterative procedure. Thereby, the finite element method (FEM) serves to calculate numerically the three-dimensional strain field within mesoscopic objects of most different shapes and various chemical compositions. However, this method remains restricted to some $10^{6}$ nodes and hence an intermediate refinement procedure becomes necessary where the strain field will be extrapolated on a regular grid with an inter-node distance of typically four times the lattice constant of silicon. Subsequently, this deformation field serves as input for a kinematical scattering routine where the scattering amplitudes arising from all participating scatterers are summed up in a coherent way. Usually only those scatterers residing within the island are taken into account in order to avoid scattering artefacts due to the finite size effect. In general, the diffusely scattered amplitude $A_{\mathrm{d}}$ can be calculated as follows:

$A_{\mathrm{d}}(\boldsymbol{q}) \propto \sum_{i} \sum_{k} \varrho\left(\boldsymbol{r}_{i, k}\right) \exp \left[\mathrm{i} \boldsymbol{q}\left(\boldsymbol{r}_{i, k}+\boldsymbol{u}\left(\boldsymbol{r}_{i, k}\right)\right)\right]$,

with $\boldsymbol{r}_{i, k}=\boldsymbol{R}_{i}+\boldsymbol{r}_{k}, \varrho$ is the electron density of the crystal lattice, $\boldsymbol{R}_{i}$ gives the position of the $i$ th supercell, $\boldsymbol{r}_{k}$ denotes the positions of the $k$ th atom within the unit cell and $\boldsymbol{u}$ denotes the displacement vector.

\section{Results and discussion}

Generally, the SiGe islands can elastically relax during the growth process and hence an incorporation of a higher germanium content, respectively unit cells with a larger lattice constant, becomes energetically favorable towards the islands apex. However, there are a variety of different conceivable germanium profiles which will fulfil this obvious energetic condition, e.g. a linear concentration gradient in growth direction, a discrete concentration step at a certain island height or a combination of a linear gradient and a homogeneous part.

The distributions in Fig. 1 depict the measured (a) and simulated (b) intensities around the symmetrical 004

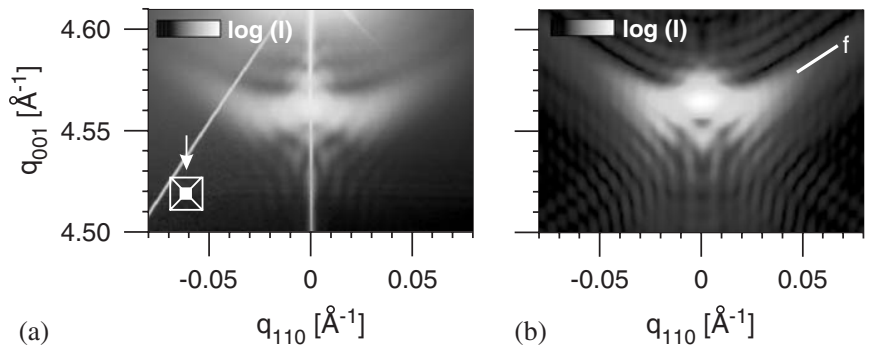

Fig. 1. Experimental (a) and simulated (b) intensity distributions around the symmetric 004 reflection within the $\left[\begin{array}{lll}1 & 1 & 0\end{array}\right]$ zone referring to a scattering plane containing the island base.

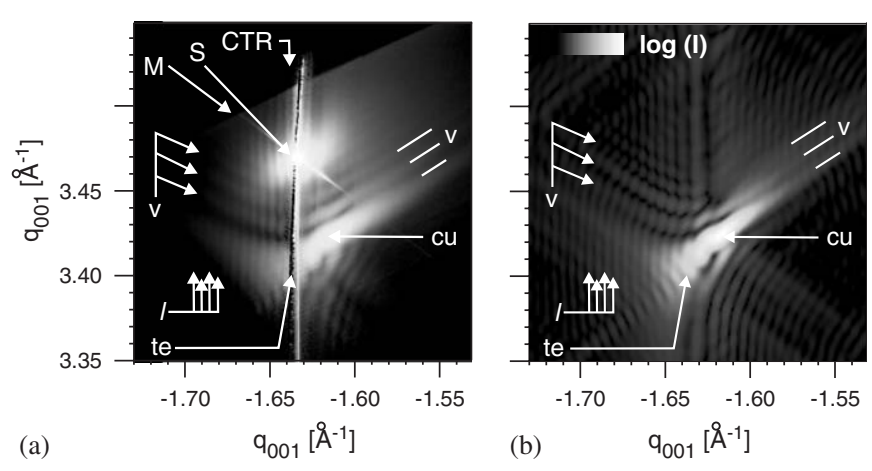

Fig. 2. Both distributions show the diffusely scattered intensity around the asymmetrical 113 reflection as measured (a) and simulated (b).

reflection within the $\left[\begin{array}{lll}1 & 1 & 0\end{array}\right]$ zone. All scattering simulations discussed here were done for the same island morphology (not shown here) which consists of a truncated pyramid confined by $\left\{\begin{array}{lll}1 & 1 & 1\end{array}\right\}$ side and an $(001)$ top facet with base width and height of 130 and $65 \mathrm{~nm}$, respectively. The island is placed on a $2 \mathrm{~nm}$ wetting layer on top of an extended $260 \mathrm{~nm} \times 260 \mathrm{~nm} \times 100 \mathrm{~nm}$ silicon substrate.

The actual substrate peak has been omitted. It appears outside the depicted area at $q_{001}=4.628 \AA^{-1}$. On the other hand, the butterfly-shaped pattern at smaller $q_{001}$ is influenced by the islands themselves and in particular by elastic strain and thus indirectly (as we will show later) serves as a fingerprint of the chemical profile. Within the present plane it is mainly dominated by the particular island shape resulting in facet rods (f) which are inclined by approximately $54.7^{\circ}$. Due to the finite size effect extended facets in real space, e.g. the $\left\{\begin{array}{lll}1 & 1 & 1\end{array}\right\}$-type island facets, cause intense features which appear in reciprocal space perpendicular to the respective planes in real space. Moreover, those facet rods are superimposed by size oscillations whose lateral period inversely scales with the island size.

Since lateral and vertical strain tensor components are not separable in a symmetric geometry an asymmetrical reflection as e.g. Si(113), Fig. 2, allows to distinguish between scattering from tetragonally distorted unit cells (te) which figure at the island bottom and scattering by a cubic lattice (cu) at the island apex where the elastic impact of the substrate almost vanishes. However, there is also a prominent contribution due to shape resulting in lateral 

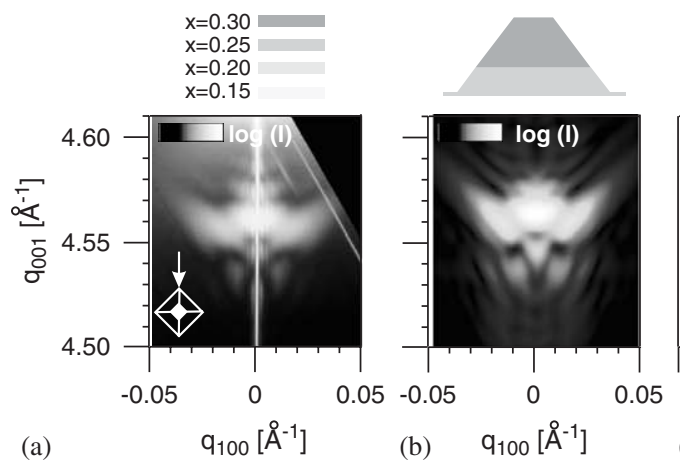

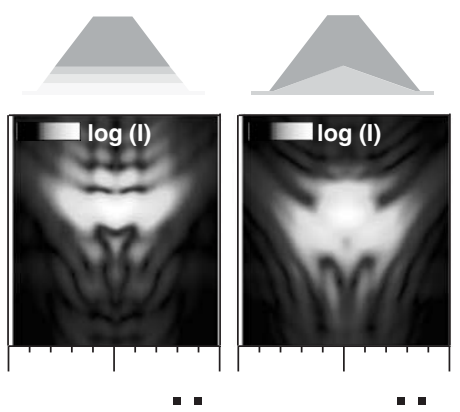

(c)

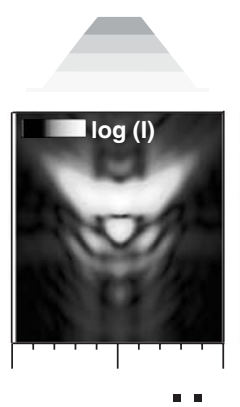

(e)

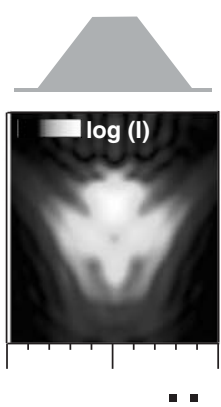

(f)

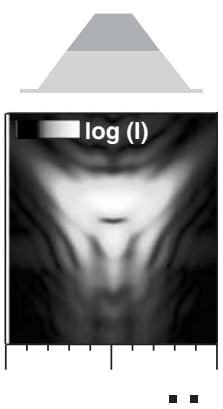

(g)

Fig. 3. (a) Shows the measured intensity distributions around the symmetric 004 reflection along the [100] direction, and, in comparison scattering simulations (b-g) on the base of various chemical profiles as depicted.

(l) and vertical (v) size oscillations. A highly intense crystal truncation $\operatorname{rod}(\mathrm{CTR})$ and the monochromator artefact (M) intersect the $\mathrm{Si}\left(\begin{array}{llll}1 & 1 & 3\end{array}\right)$ substrate reflection (S).

Suitable candidates to probe strain and hence chemical composition with a minimum shape influence are crystallographic zones where no or only small island facets are present. Fig. 3(a) shows the measured intensity distribution in the $\left[\begin{array}{lll}1 & 0 & 0\end{array}\right]$ plane. Except the intense CTR along $q_{001}$ there are no size oscillations as in Fig. 1. Corresponding simulations (b)-(g) probe the influence of strain and chemical composition as sketched in the schemes above.

Further it allows to estimate the spatial and chemical resolution. The overall shape of the diffuse scattering on the very first model, e.g. considering a homogeneous germanium content of $30 \%$ (f) clearly differs from the experiment and appears at comparatively small $q_{001}$, indicating that the averaged germanium content has to be decreased in the model. Thus, the remaining simulations prove a vertical shift of the diffuse intensity towards the silicon reflection. The most appropriate approximation (b) results from an island with a discrete concentration step at $\mathrm{h} / 3$ from $25 \%$ germanium at the island bottom towards $30 \%$ above, whereas a linear gradient (e) - approximated by four equally spaced slices of $15 \%, 20 \%, 25 \%$ and $30 \%$ does not meet the measurement. Thus, we have conceived additional models with discrete concentration steps at $\mathrm{h} / 3$ separating a linear gradient at the island bottom and a homogeneous region above (c) or considering an incorporated pyramid (d). Both models show a slightly worse agreement with respect to simulation (b). Finally, to estimate the spatial resolution in vertical direction we have shifted the interface $(25 / 30) \%$ from $\mathrm{h} / 3$ (model b) towards $\mathrm{h} / 2$ (model g) which clearly rejects the latter ones and results in an accuracy of about $5 \mathrm{~nm}$ for the interface position.

The simulations in Fig. 3 have been performed taking the different scattering abilities of germanium and silicon into account which enter the scattering process via the electron densities in Eq. (1). Since the X-ray diffraction was performed at an X-ray energy of $8 \mathrm{keV}$ which is far away from relevant absorption edges, the corresponding atomic numbers $Z_{i}$ equal the absolute value of the respective

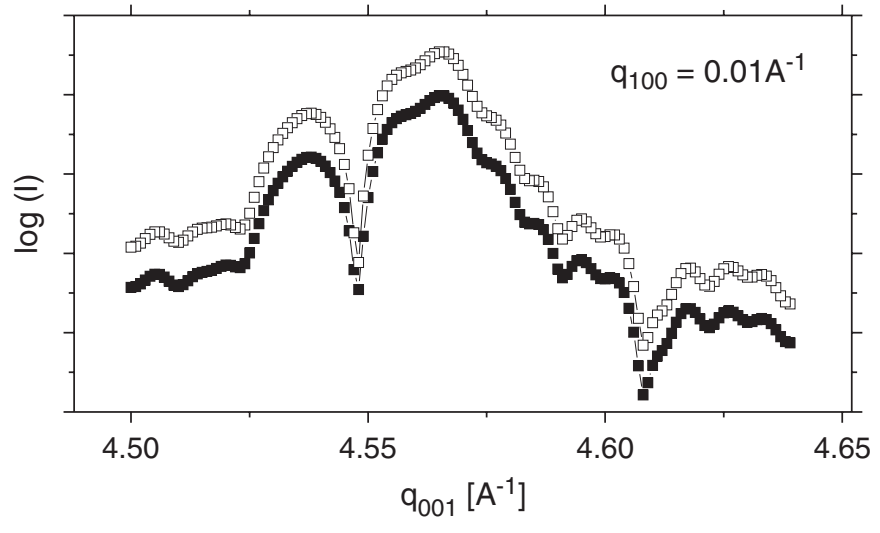

Fig. 4. Simulated vertical sections through the distributions Fig. 3(a) and (b) at a constant $q_{100}=0.01 \AA^{-1}$ taking the appropriate form factors of silicon and germanium into account (open squares) and assuming equivalent scattering abilities (full squares). Both profiles have been vertically shifted by a factor of 5 for better clarity.

structure amplitudes, and consequently there is a more intense contribution to the scattering signal out of areas with a higher germanium content (island apex), whereas the island bottom with a smaller germanium content less contributes to scattering. Assuming only two regions with different concentrations, we can define a factor which describes the ratio of both form factors:

$a=\left(\frac{x_{1} Z_{\mathrm{ge}}+\left(1-x_{1}\right) Z_{\mathrm{si}}}{x_{2} Z_{\mathrm{ge}}+\left(1-x_{2}\right) Z_{\mathrm{si}}}\right)^{2}$

which numerically results in a value of 1.08 for $x_{1}=0.25$ and $x_{1}=0.3$ as it was assumed for model Fig. 3(b). Up to this point it is still open which parameter in Eq. (1) dominates the scattering process, the displacement field and/or the chemical profile. Therefore, we have exemplarily performed a simulation similar to that shown Fig. 3(b), however, without any direct chemical influence which was done by having the scattering abilities of germanium and silicon on the same level. Since the overall distribution (not shown) looks the same as Fig. 3(b) two equivalent sections through both distributions at $q_{100}$ are shown in Fig. 4. Both are vertically shifted with respect to each other, however, 
no difference was identified. This observation clearly proves the interesting fact that a chemical profile within $\mathrm{SiGe} / \mathrm{Si}$ islands influences the diffuse scattering only by the resulting strain distribution and not directly through the different structure factors of silicon and germanium.

We have investigated the diffuse X-ray scattering of pseudomorphic $\mathrm{SiGe} / \mathrm{Si}\left(\begin{array}{ll}0 & 0\end{array}\right)$ Stranski-Krastanov islands which have been grown by means of liquid phase epitaxy. Respective scattering simulations on the base of finite element calculations for the elastic strain distribution prove a discrete horizontal concentration step at $\mathrm{h} / 3$. Comparing simulations taking the various scattering abilities of germanium and silicon into account and, on the other hand, assuming same form factors for both materials, clearly demonstrate that elastic strain dominates the diffuse scattering at X-ray energies far away from absorption edges. Consequently, the chemical profile influences the scattering process almost exclusively via a modified strain.

\section{Acknowledgments}

The authors thank W. Drube, BW2 at HASYLAB, Hamburg for experimental assistance. Discussions with M. Schmidbauer and R. Köhler, Humboldt-Universität zu Berlin are appreciated.

\section{References}

[1] V.A. Shchukin, et al., Epitaxy of Nanostructures, Springer, New York, 2004.

[2] W. Dorsch, et al., Appl. Phys. Lett. 72 (1998) 179.

[3] M. Schmidbauer, X-Ray Diffuse Scattering from Self-Organized Mesoscopic Semiconductor Structures, Springer, Berlin, 2004.

[4] J. Stangl, et al., Rev. Mod. Phys. 76 (2004) 725.

[5] U. Pietsch, et al., High Resolution X-Ray Scattering from Thin Solid Films to Lateral Nanostructures, Springer, Berlin, 2004.

[6] M. Hanke, et al., Appl. Phys. Lett. 84 (2004) 5228.

[7] M. Meixner, et al., Phys. Rev. B 64 (2001) 245307. 